\title{
Fortalezas de carácter en pacientes adultos en tratamiento psicoterapéutico
}

\section{Character strengths in adult patients in psychotherapeutic treatment}

\author{
Vanesa C. Góngora ${ }^{1}$ \\ ${ }^{1}$ Doctora en Psicología. Investigadora adjunta del Consejo Nacional de Investigaciones \\ Científicas y Técnicas (CONICET) en la Universidad de Palermo. Profesora Asociada, Facultad \\ de Ciencias Sociales, Universidad de Palermo. Buenos Aires, Argentina. \\ E-mail: gongoravanesa@gmail.com
}

\begin{abstract}
Este proyecto ha sido financiado por el Consejo Nacional de Investigaciones Científicas y Técnicas (CONICET), Buenos Aires, Argentina.

Universidad de Palermo.

Consejo Nacional de Investigaciones Científicas y Técnicas (CONICET).

Buenos Aires, Argentina.
\end{abstract}

\section{Resumen}

La investigación que se informa tuvo como objetivos: 1) estudiar las 24 fortalezas de carácter en pacientes adultos que se encuentran en diferentes etapas de un tratamiento cognitivo conductual psicoterapéutico naturalista; y 2) analizar la relación de las fortalezas con algunas del progreso durante el tratamiento, la alianza terapéutica y la adherencia al tratamiento desde la perspectiva del terapeuta. Se contó con una muestra intencional de 85 pacientes adultos de ambos sexos en tratamiento psicoterapéutico ambulatorio $\mathrm{y}$ sus respectivos terapeutas. Se utilizaron: una hoja de datos demográficos y del tratamiento, el Inventario de Fortalezas y Virtudes y una encuesta de opinión para el terapeuta. Los resultados mostraron que los pacientes en la etapa final presentan una mayor fortaleza de Autorregulación. Los pacientes con depresión presentaban menores fortalezas de apertura mental y capacidad de perdonar, en comparación con pacientes con trastornos de ansiedad y con trastornos comórbidos ansioso-depresivos. Aquellos pacientes que habían tenido un tratamiento psiquiátrico previo presentaban menores niveles de la fortaleza persistencia. Un mayor tiempo de tratamiento se asoció con mayores niveles de las fortalezas Liderazgo y curiosidad, en tanto que un mayor malestar psicológico se vinculó con una disminución de una alta cantidad de fortalezas. La fortaleza espiritualidad se halló significativamente asociada al progreso en el tratamiento según su terapeuta. Una mayor apertura mental se halló vinculada a mayor adherencia a horarios, sugerencias y mejor vínculo terapéutico según la opinión del terapeuta. El estudio del funcionamiento positivo en pacientes permitirá enriquecer la psicología clínica para convertirse en una disciplina más integradora.

Palabras clave: Psicología positiva; Pacientes; Tratamiento; Fortalezas; Adultos

\begin{abstract}
The study of positive traits or strengths has been the cornerstone of positive psychology. Positive psychology assumes that positive variables are different in nature from psychopathological variables and they do not neces-
\end{abstract}


sarily improve with a usual psychotherapeutic treatment. They would require another type of intervention to progress: a positive intervention. Studies on character strengths in the clinical population are very scarce and, in general, focused on depression. Previous studies found that higher levels of hope, vitality, spirituality and leadership strengths were related to lower levels of anxiety and depression. In addition, lower levels of gratitude have been linked to symptoms of depression. The study of strengths and progress during treatment is very limited, whereas the relationship between strengths of character and treatment process has not yet been investigated. This research aims to: 1) study the 24 character strengths in adult patients who are at different stages of a naturalistic cognitive behavioral treatment; and 2) to analyze the relationship of character strengths with progress during treatment, therapeutic alliance and adherence to treatment from the therapist's perspective. Therapist's report has proven to be a very valuable source of information about the therapeutic process and outcome. An intentional sample of 85 adult outpatients (25 men and 60 women) was used and their respective therapists (11 men and 7 women). Patients had mainly anxiety, depressive or comorbid anxiety-depressive disorders; 22 were in the initial stage, 46 in the intermediate stage and 17 in the final stage of treatment. They completed a demographic and treatment datasheet, the Inventory of Strengths and Virtues and the therapist filled in an opinion survey. The study was cross-sectional. The treatment was cognitive-behavioral. The therapists did not receive any training or instruction during study, the treatment was "as usual". Stages of treatment, initial, intermediate or final, were assigned by the therapist. The results showed that patients in the final stage have greater self-regulation strength. In this strength, there are no differences between patients at initial and intermediate stages of treatment but it increases significantly in the group that was finishing treatment. It should also be noted that in the 23 remaining strengths there are no differences among patients at the different stages of treatment. Patients with depression had lower strengths of open-mindedness and forgiveness compared to patients with anxiety disorders and comorbid anxiety-depressive disorders. Those patients who had had a previous psychiatric treatment had lower levels of Persistence strength. However, no differences were found between patients who had a previous psychological treatment and those who had not. A longer duration of treatment was associated with higher levels of leadership and curiosity strengths, while greater psychological distress was associated with a decrease in a high number of strengths. The strength spirituality was found to be significantly associated with progress in treatment according to the therapist. A greater open-mindedness was associated with better adherence to schedules, suggestions and therapeutic alliance according to the therapist's opinion. The association between character strengths and the therapist's opinion has been mild to moderate, which is expected for being a hetero-report. This has been the first study to compare the character strengths between patients at different stages of a naturalistic psychotherapeutic treatment and to include patients with anxiety disorders. This research provided a first approach to the relationship between character strengths and the therapist's perception of progress in therapy and some treatment process related variables. The study of positive functioning in patients will enrich clinical psychology to become a more integrative discipline. In this way, the goal of therapy will not only be to alleviate the negative symptoms, but also to help individuals build a full life.

Keywords: Positive psychology; Patients; Treatment; Strengths; Adults 


\section{Introducción}

\section{Variables positivas y fortalezas de carácter en pacientes adultos en tratamiento psicoterapéutico}

El estudio de los rasgos positivos o fortalezas ha sido la piedra angular de la psicología positiva (Park y Peterson, 2009). Peterson y Seligman (2004) se basaron en las tradiciones filosóficas y religiosas de Oriente y Occidente para identificar seis grandes virtudes morales: el coraje, la justicia, la humanidad, la templanza, la sabiduría y la trascendencia. A su vez, cada una de estas virtudes se asociaba con un determinado número de 24 fortalezas de carácter.

Las investigaciones realizadas con muestras de diversos países no han coincidido sobre qué fortalezas se asociaban con determinadas virtudes, ni en cuántas virtudes se organizaban las fortalezas. Los estudios han dado cuenta de 3 a 5 virtudes, por lo que hay un gran cuestionamiento a la clasificación original de seis virtudes de Peterson (Azañedo, Fernández-Abascal y Barraca, 2014; Cosentino Castro Solano, 2012; Macdonald, Bore y Munro, 2008; Ruch et al., 2010). Sin embargo, las investigaciones a nivel de fortalezas siguen siendo muy desarrolladas.

En su formulación, las virtudes y fortalezas humanas se consideraron el "reverso" o la alternativa positiva del Manual Diagnóstico y Estadístico de los Trastornos Mentales -DSM- (Peterson y Seligman, 2004). Debe señalarse, de todos modos que la formulación de Peterson no se realizó como una forma de reconstruir los trastornos del DSM. Algunas de las categorías del DSM están relacionadas con las fortalezas de Peterson: por ejemplo, "Desesperación", lo opuesto a la esperanza, captura una parte importante del trastorno depresivo mayor, pero no todo el trastorno. La desesperación, en otras palabras, no subsume el Trastorno Depresivo Mayor, ni pretende hacerlo (Seligman, 2015).

Esta postura, que es propia de la psicología positiva, asume que las variables positivas, como son las fortalezas o el bienestar, son de naturaleza diferentes a las variables psicopatológicas (Seligman, 2002). Keyes (2005) considera que la salud mental positiva y la enfermedad mental serían dos aspectos diferentes, aunque relacionados, y la ausencia de un trastorno mental no implica la presencia de altos niveles de bienestar y rasgos positivos (Keyes, 2005). Para Keyes el despliegue de fortalezas de carácter permitirían llegar a una alta salud mental positiva o funcionamiento óptimo (Castro Solano y Cosentino, 2017; Keyes, Fredrickson y Park, 2012). Numerosos estudios empíricos internacionales han apoyado este supuesto de separación de lo positivo y lo patológico (Gilmour, 2014; Lim, Ko, Shin y Cho, 2013; Petrillo, Capone, Casoy Keyes, 2015; Yin, Hey Fu, 2013). Al ser dos aspectos diferentes, se asume que las variables positivas no necesariamente mejoran con un tratamiento clínico habitual y requerirían de otro tipo de intervención para incrementarse: una intervención positiva (Fava, 1999; Seligman, 2002).

Sin embargo, una perspectiva alternativa sostenida por la psicología clínica positiva afirma que no hay tal diferencia entre variables positivas y negativas, ya que ambas coexistirían en el mismo continuo (Wood y Tarrier, 2010). Los autores afirman que todas las variables positivas y negativas tienen una inversa (por ejemplo, la depresión frente a la felicidad, la gratitud frente a la ingratitud) y el foco de un extremo del continuo, ya sea positiva o negativa, depende del interés del psicólogo o investigador (Johnson y Wood, 2016). Al plantear un continuo positivo-negativo, esta perspectiva considera que las mismas terapias pueden funcionar para mejorar tanto el funcionamiento positivo como el negativo (Joseph y Wood, 2010), sin embargo, las investigaciones que avalan esta postura son casi inexistentes. En un estudio reciente, se encontró que no había diferencias en la eficacia para disminuir el nivel de sintomatología depresiva y aumentar el nivel 
del bienestar entre una terapia positiva y una terapia cognitivo conductual para la depresión. Ambos tipos de terapias resultaron igualmente eficaces (Chaves, López-Gómez, Hervasy Vázquez, 2016).

Los estudios que han investigado las fortalezas de carácter vinculadas a trastornos clínicos, lo han hecho mayormente sobre depresión, utilizando población general o universitaria con sintomatología significativa y en menor medida, población clínica. Mayores niveles de las fortalezas Esperanza, Vitalidad y Liderazgo encontraron relación con menores niveles de ansiedad y depresión en adolescentes (Park y Peterson, 2008). Asimismo, la Esperanza se asoció con menor malestar psicológico en este grupo de edad (Gilman, Dooley y Florell, 2006). Las fortalezas de Amabilidad, Inteligencia Social y Autorregulación se vincularon con menores efectos de trauma y estrés en población adulta (Park y Peterson, 2009). El aumento de la Esperanza se asoció con disminuciones significativas en los niveles de depresión y ansiedad en una muestra comunitaria (Cheavens, Feldman, Gum, Michael y Snyder, 2006). En un estudio de metaanálisis, mayores niveles de la fortaleza Espiritualidad se vincularon con menores niveles de depresión (Smith, McCullough y Poll, 2003). Otros estudios encontraron que la Capacidad de Perdonar disminuía el impacto del malestar psicológico evaluado como depresión, ansiedad y estrés en estudiantes universitarios (Orcutt, 2006). Se encontró que la Autenticidad amortiguaba la asociación entre bajo apoyo social y síntomas depresivos en adolescentes (Theran, 2010). En un estudio retrospectivo, aquellas personas que se habían recuperado de una historia de trastornos psicológicos mostraron mayores fortalezas de Apreciación de la Belleza, Creatividad, Curiosidad, Gratitud y Amor por el Conocimiento (Peterson, Park y Seligman, 2006).

Varios estudios han destacado la vinculación de la Gratitud con síntomas de depresión. El aumento de esta fortaleza a través de una intervención positiva disminuía significativa- mente los niveles de depresión en estudiantes y en pacientes depresivos (Seligman, Rashidy Parks, 2006; Seligman, Steen, Park Peterson, 2005). Asimismo, la Gratitud se asociaba a menor ideación suicida en estudiantes universitarios deprimidos (Kleiman, Adams, Kashdany Riskind, 2013). Finalmente, las fortalezas Esperanza, Apreciación por la Belleza y Espiritualidad se asociaron con la recuperación de pacientes con depresión en tratamiento cognitivo conductual más allá del papel desempeñado por las vulnerabilidades (Huta y Hawley, 2010).

La revisión de la literatura indica que los estudios sobre fortalezas en población en tratamiento psicoterapéutico son muy escasos y en general, centrados en depresión. Se hace necesario incluir otro tipo de pacientes como aquellos con trastornos ansiosos para ampliar el espectro clínico. Asimismo, no hay estudios que hayan comparado fortalezas de carácter en pacientes que se encontraban en distintas etapas de tratamiento psicoterapéutico, así como su relación con la cronicidad de la patología (duración de la enfermedad, tratamientos previos).

El primer objetivo de la presente investigación fue estudiar las 24 fortalezas de carácter en pacientes adultos que se encontraban en diferentes etapas de un tratamiento cognitivo conductual naturalista. Más específicamente, se planteó comparar las fortalezas en pacientes según etapa de tratamiento en la que se encontraba (inicial / intermedia / final), tipo de sintomatología predominante (por ejemplo,. ansioso, depresivo, mixta), existencia de tratamiento psicológico o psiquiátrico previo, así como su relación con el nivel de malestar psicológico, duración de la enfermedad y tiempo de tratamiento.

Se esperaba que predominara un nivel más bajo de fortalezas en la fase inicial del tratamiento en comparación con la fase final. Los pacientes con sintomatología predominantemente depresiva tendrían menores niveles de fortalezas que el resto de los pacientes. Se esperaba un menor nivel de fortalezas en 
pacientes con mayor malestar psicológico, con mayor duración de la enfermedad y con existencia de tratamiento previo.

También resultó de interés, al estudiar fortalezas de carácter en pacientes en tratamiento, su relación con el progreso durante la terapia y con variables de proceso terapéutico. Desde la psicología positiva se sostiene que bajos niveles de variables positivas, en este caso fortalezas de carácter, tienden a tener trastornos más crónicos y menos progreso en el tratamiento que aquellos con niveles altos (Huta y Hawley, 2010; Keyes, 2007). El estudio sobre fortalezas y el progreso durante el tratamiento es muy escaso, en tanto que la relación entre fortalezas de carácter y variables relacionadas con el proceso de tratamiento aún no ha sido investigada.

Un segundo objetivo es estudiar las fortalezas de carácter en relación con algunas variables de tratamiento como progreso durante el tratamiento, alianza terapéutica y la adherencia al tratamiento desde la perspectiva del terapeuta.

El informe del terapeuta ha demostrado ser una fuente muy valiosa de información de proceso terapéutico, ya que es una fuente diferente y complementaria a la provista por el paciente. Los reportes de los terapeutas también son valiosos para entender los factores comunes o no específicos de psicoterapia tales como la alianza terapéutica y el compromiso del paciente (Bachelor, 2013; Clemence, Hilsenroth, Ackerman, Strassle y Handler, 2005; Laska, Gurman y Wampold, 2014). Laska (2014) examinó las informaciones provistas por el terapeuta y por el paciente sobre la alianza terapéutica, y fue el aporte del terapeuta, más que el del paciente, el que mejor predijo los resultado de la terapia. Asimismo, la percepción del terapeuta sobre el compromiso y la participación activa de los pacientes se encontró asociada a mejores resultados de la terapia (Bachelor, 2013).

Se espera que los pacientes con fortalezas de carácter más elevadas sean percibidos con mayor progreso terapéutico por el terapeuta.
Además, aquellos pacientes con mayores niveles de fortalezas serán percibidos con una mejor adherencia al tratamiento y un mejor vínculo con su terapeuta.

\section{Metodología}

\section{Muestra}

\section{Pacientes}

Se seleccionó una muestra intencional de 85 pacientes de ambos sexos (25 hombres y 60 mujeres) en tratamiento psicoterapéutico ambulatorio en centros o servicios de salud mental de la ciudad de Buenos Aires, cuyas edades oscilaban entre 18 y 75 años $(\mathrm{M}=40.83, \mathrm{DE}=16.0)$. Se excluyeron a pacientes internados, pacientes con diagnóstico de esquizofrenia y otros estados psicóticos, demencias y trastornos cognoscitivos y trastornos bipolares, así como aquellos cuyo estado de crisis les impedía completar los cuestionarios. En cuanto al estado civil, 45 (52.9\%) eran solteros, $23(27.1 \%)$ casados, 14 (16.5\%) divorciados y $3(3.5 \%)$ viudos. Una minoría $(23.5 \%)$ había alcanzado la educación primaria $(\mathrm{n}=20)$ como nivel máximo de estudios, $51.8 \%(\mathrm{n}=44)$ la educación media y $24.7 \%(\mathrm{n}=21)$ una educación terciaria o universitaria. Con respecto al tipo de patología, el $58.8 \%$ presentaba un trastorno de tipo ansioso, un $21.2 \%$ un trastorno de tipo depresivo, un $17.6 \%$ trastornos comórbidos con síntomas ansiosos y depresivos y un 2.4 $\%$ algún otro trastorno. En cuanto a la etapa de tratamiento, 22 pacientes se encontraban en la fase inicial, 46 en una etapa intermedia y 17 en una etapa final. La duración media de tratamiento de los participantes era de 6.75 meses $(\mathrm{DE} .=8.59, \mathrm{M}=4.5)$.

\section{Terapeutas que trataron a los pacientes participantes del estudio}

Se incluyeron 11 hombres y 7 mujeres, todos psicólogos con un mínimo de 5 años de 
experiencia clínica en el tratamiento cognitivo-conductual. Ninguno había recibido entrenamiento en intervenciones positivas.

El tratamiento fue de tipo cognitivo-conductual. Los terapeutas no recibieron ningún entrenamiento o instrucción en particular sobre el tratamiento a realizar durante esta investigación, la psicoterapia fue en forma habitual. Además de la terapia psicológica, el $62.4 \%$ de los pacientes también asistía a control psiquiátrico y recibía medicación (principalmente antidepresivos y ansiolíticos).

\section{Instrumentos}

\section{Inventario de Fortalezas y Virtudes (IV y Fabre)}

Este instrumento fue diseñado tomando en cuenta el modelo de fortalezas y virtudes de Peterson y Seligman (2004). Consta de 24 ítems que se evalúan con 5 opciones de respuesta que van de: muy diferente a mí (1) a muy parecido a mí (5). Cada uno de los ítems evalúa una fortaleza diferente. También se pueden calcular las dimensiones correspondientes a las diferentes virtudes evaluadas por el instrumento. La escala ha sido desarrollada y validada en población general adulta y en población militar argentina (Cosentino, 2009; Cosentino y Castro Solano, 2012). La fiabilidad total de la escala en la muestra clínica fue $\alpha=.89$.

\section{Hoja de datos generales del paciente y del tratamiento}

Se indagó acerca de edad, sexo, nivel educativo, estado civil, motivo de consulta, tiempo de tratamiento, etapa del tratamiento (inicial / intermedia / final) y diagnóstico principal. La etapa inicial corresponde a las primeras sesiones de tratamiento en las que se evalúan los síntomas del paciente, se formula el diagnóstico, se inicia la relación terapéutica y se establecen los objetivos y estrategias de tratamiento. La etapa intermedia es en la que se desarrolla propiamente el tratamiento. El terapeuta emplea técnicas y estrategias terapéuticas sobre la base de los objetivos del tratamiento. Es la parte más larga y principal del tratamiento. La etapa final se considera cuando los objetivos terapéuticos están prácticamente cumplidos, los síntomas remiten y se está trabajando en el alta del tratamiento. Se supone que la duración de las etapas depende de las características de los pacientes; por ejemplo, algunos clientes necesitarán más tiempo de evaluación, mientras que otros, menos.

\section{Encuesta de opinión sobre el progreso del paciente, adherencia al tratamiento y relación terapéutica}

Se solicitó a los terapeutas completar cinco ítems sobre sus pacientes. Dos ítems se referían al progreso que consideraban los pacientes desde el inicio del tratamiento (progreso en relación al motivo de consulta $\mathrm{y}$ progreso en relación con los principales síntomas). Se evaluaron en una escala de Likert de 5 opciones (peor, igual, un poco mejor, mucho mejor, excelente progreso). Otros dos ítems se referían a la adherencia al tratamiento: cumplimiento de horarios y asistencia a las sesiones, y cumplimiento de sugerencias o indicaciones del terapeuta (malo, regular, bueno, muy bueno, excelente). Un último ítem sondeó la opinión del terapeuta sobre la relación terapéutica (mala, regular, buena, muy buena, excelente).

\section{Procedimiento}

Se solicitó a los terapeutas que seleccionaran a pacientes en distintas etapas de tratamiento, que pudieran participar en el estudio conforme a los criterios de inclusión y exclusión mencionados en el apartado Muestra. Los pacientes fueron informados de los objetivos de la investigación y completaron un consentimiento informado. La administración de las pruebas se realizó en forma individual, durante 
una única sesión de 45 minutos en presencia de un miembro del equipo de investigación. El terapeuta completó la hoja con datos y evaluación de su opinión sobre el progreso del paciente desde el inicio del tratamiento en una única sesión de 5 minutos.

\section{Análisis de datos}

En primer lugar, se efectuaron una serie de análisis de varianza Anova-Oneway con análisis post-hoc de Tukey para comparar las 24 fortalezas de carácter entre distintas variables independientes: etapa del tratamiento (inicial / intermedia / final) y tipo diagnóstico principal (depresión / ansiedad / mixto). Posteriormente, se utilizó la prueba $t$ para comparar las 24 fortalezas entre pacientes con y sin tratamiento psiquiátrico previo, y entre pacientes con y sin tratamiento psicológico previo. Asimismo, se realizaron análisis de correlación de Pearson entre las 24 fortalezas y el tiempo de tratamiento, la duración de la enfermedad y el nivel de malestar general del paciente. Finalmente, se correlacionaron las fortalezas de carácter con el autorreporte del terapeuta: percepción del progreso del paciente, percepción de adherencia al tratamiento y percepción del vínculo terapéutico.

\section{Resultados}

En primer lugar se realizó una AnovaOneway para comparar las 24 fortalezas de carácter entre los pacientes que estaban en las tres etapas de tratamiento (inicial / intermedia / final). Los resultados del análisis indicaron que la fortaleza Autorregulación $(\mathrm{F}=4.92, \mathrm{p}$ $\left.=.01, \eta^{2}=.11\right)$ mostró diferencias estadísticamente significativas, con un tamaño de efecto grande. El análisis post-hoc de Tukey indicó que esta fortaleza era más alta en el grupo que se encontraba en la etapa final con respecto a los otros dos grupos.

El mismo procedimiento se utilizó para comparar las 24 fortalezas de acuerdo al tipo diagnóstico principal. Dado que había un grupo de solo 2 pacientes en Otros diagnósticos, se decidió eliminar este grupo para este análisis a los fines de evitar sesgos. Los grupos de diagnóstico quedaron conformados de la siguiente manera: trastornos ansiosos $(\mathrm{n}=50)$, trastornos depresivos $(\mathrm{n}=18) \mathrm{y}$ trastornos comórbidos ansioso-depresivo (n =15). El análisis mostró que dos fortalezas presentaban diferencias significativas entre los grupos: Apertura mental $(\mathrm{F}=6.41, \mathrm{p} \leq .01$, $\left.\eta^{2}=.14\right)$ y Capacidad de perdonar $(\mathrm{F}=3.48, \mathrm{p}$ $\left.=.03, \eta^{2}=.08\right)$. En el primer caso, el tamaño de efecto de las diferencias fue grande y en el segundo, moderado. El grupo de pacientes con trastornos depresivos mostraron menores niveles de ambas fortalezas en tanto que el grupo con trastornos comórbido ansioso-depresivos evidenciaron mayores niveles en estas variables.

Posteriormente, se compararon las fortalezas de carácter entre aquellos pacientes que habían tenido un tratamiento psiquiátrico previo $(n=30)$ y quienes no $(n=55)$. La prueba $t$ de comparación de medias indicó que aquellos que habían tenido un tratamiento previo presentaban significativamente menores niveles de la fortaleza Persistencia $(t=2.91$, $p<.01, \mathrm{~d}=-.73)$ en relación al otro grupo. El tamaño de la diferencia fue de moderado a alto. No se encontraron diferencias significativas en ninguna fortaleza entre los pacientes que habían realizado tratamiento psicológico previo $(n=63)$ y los que no habían realizado uno $(\mathrm{n}=22)$.

Se realizaron análisis de correlación entre las 24 fortalezas y el tiempo de tratamiento. Se halló que, a mayor tiempo de tratamiento, mayor nivel de la fortaleza Liderazgo $(r=.24$, $\mathrm{p}=.03$ ) y mayor nivel de la fortaleza Curio$\operatorname{sidad}(\mathrm{r}=.26, \mathrm{p}=.02)$. No se encontró ninguna asociación significativa entre las fortalezas de carácter y la duración de la enfermedad.

Con respecto al nivel de malestar general, se encontraron 15 de las 24 asociaciones posibles en forma significativa. Las correlaciones se presentan en la Tabla 1. Se verifica una asociación negativa entre las fortalezas y el 
malestar con un tamaño de efecto de moderado a fuerte. Las correlaciones más importantes se observaron con las fortalezas Vitalidad ( $\mathrm{r}$
$=-.53)$, Curiosidad $(\mathrm{r}=.49)$, Liderazgo $(\mathrm{r}=$ -.44), Humor $(\mathrm{r}=-.40)$ y Autorregulación $(\mathrm{r}$ $=.40)$.

\section{Tabla 1}

Análisis de correlación entre las fortalezas de carácter y el nivel de malestar psicológico en los pacientes $(N=85)$.

\begin{tabular}{c|c} 
Fortaleza & Malestar Psicológico \\
\hline Apreciación de la belleza y la excelencia & -.03 \\
Imparcialidad & -.13 \\
Persistencia & $-.28^{* *}$ \\
Creatividad & .04 \\
Amor & $-.28^{* *}$ \\
Autorregulación & $-.40^{* * *}$ \\
Gratitud & $-.30^{* *}$ \\
Liderazgo & $-.44^{* * *}$ \\
Apertura mental & $-.23^{*}$ \\
Inteligencia social & $-.29^{* *}$ \\
Capacidad de Perdonar & -.09 \\
Espiritualidad & .07 \\
Trabajo en equipo & $-.29^{* *}$ \\
Valentía & $-.23^{*}$ \\
Curiosidad & $-.49^{* * *}$ \\
Bondad & -.03 \\
Esperanza & $-.31^{* *}$ \\
Integridad & $-.32^{* *}$ \\
Perspectiva & -.16 \\
Prudencia & $-.29^{* *}$ \\
Humor & $-.40^{* * *}$ \\
Humildad & -.14 \\
Amor por el saber & -.03 \\
Vitalidad & $-.53^{* * *}$ \\
.001 & \\
&
\end{tabular}

Notas: $* \mathrm{p}<.05 / * * \mathrm{p}<.01 / * * * \mathrm{p}<.001$.

En cuanto a la asociación entre las fortalezas de carácter y la percepción del terapeuta sobre el progreso del paciente desde el ingreso a tratamiento, se encontró que la fortaleza Espiritualidad se halló significativamente asociada al progreso en relación al motivo de consulta $(\mathrm{r}$ $=.27, \mathrm{p}=.01)$ y en relación a la sintomatología inicial $(\mathrm{r}=.23, \mathrm{p}=.04)$.

En lo que se refiere a los factores inespecíficos del tratamiento, un mayor nivel de la fortaleza Apertura mental se encontró significativa correlacionada con una mayor percepción de adherencia al tratamiento por asistencia a sesiones y cumplimiento de horarios $(\mathrm{r}=.28$, $\mathrm{p}=.01)$ y a mayor percepción de cumplimiento de sugerencias o indicaciones del terapeuta $(\mathrm{r}=$ $.24, \mathrm{p}=.02)$. Por último, un mejor vínculo terapéutico a opinión del terapeuta se asoció con mayor nivel de las fortalezas Apertura mental $(\mathrm{r}=.29, \mathrm{p}<.01)$ y Humor $(\mathrm{r}=.22, \mathrm{p}=.04)$. 


\section{Discusión}

Los resultados de este estudio mostraron que los pacientes adultos que se encuentran en la etapa final de un tratamiento psicoterapéutico presentan una mayor fortaleza de Autorregulación. En esta fortaleza, no se observan diferencias entre etapa inicial e intermedia, pero aumenta significativamente en el grupo que está finalizando su tratamiento. Esta fortaleza implica la regulación o control de emociones y conductas, y una mayor adaptación a las situaciones cotidianas (Peterson, 2006). Resulta lógico que pacientes que han transcurrido buena parte de su tratamiento y se encuentran próximos a ser dados de alta, tengan una mayor autorregulación emocional que los que están con una mayor sintomatología. Particularmente, hay que tener en cuenta que los pacientes de la muestra ingresaron con trastornos depresivos y ansiosos, con lo cual la autorregulación emocional y conductual es esperable en una etapa final de tratamiento (Brinkmann y Franzen, 2015; Cisler, Olatunji, Feldner y Forsyth, 2010).

Si bien este resultado es coherente con la hipótesis planteada en este estudio, debe señalarse también que en las 23 fortalezas restantes no se observan diferencias entre los pacientes en distintas etapas del tratamiento. El hecho de que sea una sola fortaleza parecería más avalar la hipótesis de la psicología positiva en cuanto a que las variables positivas no aumentan necesariamente en un tratamiento común, sino que requerirían un tratamiento específico: una terapia positiva (Seligman, et al., 2006). La característica transversal del estudio no permite conocer cómo eran las fortalezas del paciente al ingreso al tratamiento y evaluar su evolución . Para ello sería necesario realizar un estudio longitudinal de seguimiento.

En cuanto a la comparación de las fortalezas según el diagnóstico principal de los pacientes, se encontró que aquellos con depresión presentaban menores fortalezas de Apertura Mental (pensamiento crítico) y Capacidad de Perdonar. Un bajo nivel en ciertas fortalezas ha sido sistemáticamente reportado en estudios con pacientes deprimidos (Cheavens, et al., 2006; Orcutt, 2006; Park y Peterson, 2008; Seligman, et al., 2006; Smith, et al., 2003; Theran, 2010). Este estudio ha incluido por primera vez, otras patologías como los trastornos de ansiedad y patologías comórbidas ansioso-depresivas. Justamente este último grupo ha sido el que evidenció fortalezas más altas. Resulta difícil explicar por qué pacientes con comorbilidades tienen más fortalezas que pacientes con cuadros simples. Una hipótesis podría ser que al ampliar el tipo de síntomas y que los cuadros clínicos sean menos puros se vean menos afectadas algunas fortalezas de carácter. De todos modos, son necesarios más estudios con distintos grupos clínicos para confirmar estos resultados.

Aquellos pacientes que habían asistido previamente a tratamiento psiquiátrico mostraron menos fortaleza de Persistencia que aquellos que no habían asistido. Se podría pensar que una baja Persistencia justamente se asocia con la falta de adherencia a un tratamiento psiquiátrico. Seligman (2015) afirma que lo contrario a la persistencia sería la indefensión, una característica que se asocia con alta sintomatología y bajo nivel de afrontamiento. Sin embargo, no se encontró diferencia entre pacientes con y sin tratamiento psicológico previo. Esto se puede deber a la alta frecuencia $(74 \%)$ de pacientes con tratamiento previo. Debe señalarse que un alto porcentaje de personas asiste al psicólogo en Argentina, no solo por sintomatología importante, sino por orientación ante problemas cotidianos. Escalante y Leiderman (2008) encontraron que el $78.1 \%$ de la población adulta de Buenos Aires había asistido al menos una vez en su vida a psicoterapia y el $15.6 \%$ lo hacía al momento del estudio (Escalante \& Leiderman, 2008). Sin embargo, la asistencia al psiquiatra supone padecer síntomas de cierta gravedad.

Un hallazgo bastante claro fue que un mayor malestar psicológico se asoció con la 
disminución de una alta cantidad de fortalezas. Un total de 15 de las 24 fortalezas se encontraron disminuidas. La disminución de fortalezas asociada a alto malestar ha sido reportada en estudios previos con adolescentes y en estudiantes universitarios deprimidos (Gilman, et al., 2006; Orcutt, 2006; Park y Peterson, 2009). En este estudio se agregaría a los pacientes en tratamiento psicoterapéutico. Esta asociación inversa apoyaría la hipótesis de la psicología clínica positiva que sostiene el continuo entre variables positivas y negativas (Joseph y Wood, 2010), es decir, al estar alto el nivel de sintomatología, se verían disminuidas las variables positivas, en este caso, las fortalezas de carácter.

Un mayor tiempo de tratamiento se asoció con más fortalezas para organizar y liderar actividades grupales (liderazgo) y mayor despliegue de inquietudes intelectuales, intereses y actividades (curiosidad). Clemence et al. (2005) también reportó que los pacientes que han estado más tiempo en terapia reportan un mejor funcionamiento general y en distintas áreas de su vida.

Una fortaleza que se encontró muy relacionada con la percepción del terapeuta sobre el paciente fue la Apertura mental. Esta fortaleza se halló vinculada a variables relacionadas al proceso terapéutico: adherencia a horarios, sugerencias y vínculo. La apertura mental o pensamiento crítico caracteriza a una persona que tiene la voluntad de buscar activamente evidencias en contra de sus creencias, planes o metas preferidas y, al hallarlas, evaluarlas de un modo justo, siendo capaz de cambiar su punto de vista, como consecuencia de esa evaluación (Peterson, 2006). El replanteo de las propias creencias y la consideración de perspectivas alternativas son parte esencial del trabajo terapéutico en las terapias cognitivo-conductuales a través de la reestructuración cognitiva (Hofmann, Asmundsony Beck, 2013), por ello es comprensible que un terapeuta relacione más esta fortaleza con la adherencia y vínculo con el paciente. De igual modo, un paciente con mayor flexibilidad cognitiva aceptará mejor las sugerencias e indicaciones de su terapeuta.

Por otro lado, la fortaleza Espiritualidad apareció muy vinculada a la percepción del terapeuta con respecto al progreso que había tenido el paciente desde que ingresó al tratamiento. Numerosos estudios han destacado el valor de esta fortaleza en relación al progreso terapéutico, particularmente en pacientes con depresión (Huta y Hawley, 2010; Smith, et al., 2003). Debe señalarse que en este estudio el progreso es desde la perspectiva del terapeuta, pero los resultados coinciden con las investigaciones previas.

También es importante mencionar que la relación entre las fortalezas y las variables de progreso y proceso terapéutico a opinión del terapeuta ha sido de leve a moderada, pero esto es explicable por ser un hétero-informe. Es esperable una menor asociación entre los hétero -informes en comparación con los auto-informes (Meyer et al., 2001). Meyer et al. (2001) encontró que las correlaciones entre el paciente y el terapeuta eran de bajas a moderadas ( $r$ entre .14 y .34 . En este estudio, las correlaciones halladas entre las fortalezas del paciente y las variables reportadas se ubican entre .22 y.29, lo que estaría indicando que, aunque relativamente bajas, son adecuadas y esperadas para este tipo de asociación.

Dentro de las limitaciones de este estudio pueden mencionarse en primer lugar, el diseño de tipo transversal que no permite hacer inferencias causales y saber cómo ingresan y cambian los pacientes durante el tratamiento. Sería importante avanzar en un estudio longitudinal para establecer el proceso de cambio de las fortalezas durante el tratamiento. En segundo lugar, debe señalarse el número limitado de pacientes. Aunque el tamaño de muestra no es muy pequeño, habría sido deseable un grupo más grande, en particular de los pacientes en las etapas inicial y final del tratamiento. En tercer lugar, se utilizó un estudio naturalizado de tratamiento o tratamiento usual. Este tipo de estudio tiene la ventaja de mostrar los datos de los pacientes 
en un entorno de tratamiento más realista (Leichsenring, 2004). El propósito de este estudio fue examinar las fortalezas de carácter en un tratamiento habitual de corte cognitivo conductual y por esa razón se eligió un estudio naturalista. Sin embargo, es importante considerar que, como el tipo de tratamiento no fue controlado, las diferencias en la implementación del tratamiento entre terapeutas podrían afectar los resultados. Por último, en relación con las variables relacionadas con el proceso de tratamiento, no se utilizaron instrumentos estandarizados para evaluar el progreso, la relación terapéutica y la adherencia al tratamiento. Un instrumento posiblemente estandarizado habría sido más válido y fiable. No obstante, los resultados son muy consistentes con investigaciones previas sobre el proceso terapéutico que han utilizado tales instrumentos, lo que nos dan cierta confianza en la adecuación de los instrumentos utilizados en este estudio. Las investigaciones futuras deberían profundizar el estudio del proceso terapéutico y las fortalezas, incluyendo también la perspectiva del paciente

Finalmente, este ha sido el primer estudio en comparar las fortalezas de carácter entre las distintas etapas de un tratamiento psicoterapéutico naturalista. También ha sido el primero en incluir pacientes con trastornos de ansiedad, grupo muy escaso en las investigaciones con fortalezas. Asimismo, esta investigación proporcionó un primer acercamiento a la relación entre las fortalezas de carácter y la percepción del terapeuta del progreso en la terapia y algunas variables relacionadas con el proceso de tratamiento. El estudio del funcionamiento positivo en pacientes permitirá enriquecer la psicología clínica para convertirse en una disciplina más integradora. De esta manera, el objetivo de la terapia no solo será aliviar el negativo, sino también ayudar a los individuos a construir una vida más plena.

\section{Referencias bibliográficas}

Azañedo, C.M., Fernández-Abascal, E.G. y
Barraca, J. (2014). Character strengths in Spain: Validation of the Values in Action Inventory of Strengths (VIA-IS) in a Spanish sample. Clínica y Salud, 25(2), 123-130. http:// dx.doi.org/10.1016/j.clysa.2014.06.002

Bachelor, A. (2013). Clients' and Therapists'Views of the Therapeutic Alliance: Similarities, Differences and Relationship to Therapy Outcome. Clinical Psychology \& Psychotherapy, 20(2), 118-135. http://dx.doi.org/10.1002/cpp.792

Brinkmann, K. y Franzen, J. (2015). Depression and Self-Regulation: A Motivational Analysis and Insights from Effort-Related Cardiovascular Reactivity. En G. H. E. Gendolla, M. Tops \& S.L. Koole (Eds.), Handbook of Biobehavioral Approaches to Self-Regulation (pp. 333-347). New York, NY: Springer New York.

Castro Solano, A. y Cosentino, A. (2017). The relationships between character strengths and life fulfillment in the view of lay-people in Argentina. Interdisciplinaria. Revista de Psicología y Ciencias Afines, 33(1), 65-80.

Cisler, J.M., Olatunji, B.O., Feldner, M.T. y Forsyth, J.P. (2010). Emotion Regulation and the Anxiety Disorders: An Integrative Review. Journal of psychopathology and behavioral assessment, 32(1), 68-82. http://dx.doi: 10.1007/s10862-009-9161-1

Clemence, A.J., Hilsenroth, M.J., Ackerman, S.J., Strassle, C.G. y Handler, L. (2005). Facets of the therapeutic alliance and perceived progress in psychotherapy: relationship between patient and therapist perspectives. Clinical Psychology \& Psychotherapy, 12(6), 443-454. http:// dx.doi: $10.1002 /$ cpp.467

Cosentino, A.C. (2009). Evaluación de las Virtudes y Fortalezas Humanas en Población de Habla Hispana. Psicodebate, 10, 53-71.

Cosentino, A.C. y Castro Solano, A. (2012). Character Strengths: A Study of Argentinean Soldiers. The Spanish Journal of Psychology, 15(1), 199-215. http://dx.doi: 10.5209/rev_ SJOP.2012.v15.n1.37310

Chaves, C., Lopez-Gomez, I., Hervas, G. y Vazquez, C. (2016). A Comparative Study on the Efficacy of a Positive Psychology Intervention and a Cognitive Behavioral Therapy for 
Clinical Depression. Cognitive Therapy and Research, 1-17. http://dx.doi: 10.1007/s10608016-9778-9

Cheavens, J.S., Feldman, D.B., Gum, A., Michael, S.T. y Snyder, C. R. (2006). Hope therapy in a community sample: A pilot investigation. [Journal; Peer Reviewed Journal]. Social Indicators Research, 77(1), 61-78.

Escalante, C. y Leiderman, E. (2008). Prevalencia de tratamiento psicoterapéutico en los habitantes de la ciudad de Buenos Aires. ERTE, 19, 261-267.

Fava, G.A. (1999). Well-being therapy: Conceptual and technical issues. Psychotherapy and Psycosomatics, 68, 171-179.

Gilman, R., Dooley, J. y Florell, D. (2006). Relative Levels of Hope and Their Relationship With Academic and Psychological Indicators Among Adolescents. Journal of Social and Clinical Psychology, 25(2), 166-178. http:// dx.doi: 10.1521/jscp.2006.25.2.166

Gilmour, H. (2014). Positive mental health and mental illness. Health reports, 25(9), 3-9.

Hofmann, S.G., Asmundson, G.J.G. y Beck, A.T. (2013). The Science of Cognitive Therapy. Behavior Therapy, 44(2), 199-212. http:// dx.doi.org/10.1016/j.beth.2009.01.007

Huta, V. y Hawley, L. (2010). Psychological Strengths and Cognitive Vulnerabilities: Are They Two Ends of the Same Continuum or Do They Have Independent Relationships with Well-being and Ill-being? [journal article]. Journal of Happiness Studies, 11(1), 71-93. http://dx.doi: 10.1007/s10902-008-9123-4

Johnson, J. y Wood, A.M. (2016). Integrating positive and clinical psychology: Viewing human functioning as continua from positive to negative can benefit clinical assessment, interventions and understandings of resilience. Cognitive Therapy and Research, 1-15.

Joseph, S. y Wood, A. (2010). Assessment of positive functioning in clinical psychology: Theoretical and practical issues. Clinical Psychology Review, 30(7), 830-838. http://dx.doi. org/10.1016/j.cpr.2010.01.002

Keyes, C.L.M.(2005). Mental illness and/or mental health? Investigating axioms of the complete state model of health. Journal of consulting and clinical psychology, 73(3), 539-548. http:// dx.doi: 10.1037/0022-006X.73.3.539

Keyes, C.L.M. (2007). Promoting and protecting mental health as flourishing: a complementary strategy for improving national mental health. The American psychologist, 62(2), 95-108. http://dx.doi: 10.1037/0003-066X.62.2.95

Keyes, C.L.M., Fredrickson, B.L. y Park, N. (2012). Positive Psychology and the Quality of Life. En K.C. Land, A.C. Michalos y M.J. Sirgy (Eds.), Handbook of Social Indicators and Quality of Life Research (pp. 99-112). Dordrecht: Springer Netherlands.

Kleiman, E.M., Adams, L.M., Kashdan, T. B. y Riskind, J.H. (2013). Grateful individuals are not suicidal: Buffering risks associated with hopelessness and depressive symptoms. Personality and Individual Differences, 55(5), 595-599. http://dx.doi.org/10.1016/j. paid.2013.05.002

Laska, K.M., Gurman, A.S. y Wampold, B.E. (2014). Expanding the lens of evidence-based practice in psychotherapy: a common factors perspective. Psychotherapy (Chicago, Ill.), 51(4), 467-481. http://dx.doi: 10.1037/ a0034332

Leichsenring, F. (2004). Randomized controlled versus naturalistic studies: A new research agenda. Bulletin of the Menninger Clinic, 68(2), 137-151. http://dx.doi.org/ 10.1521/ bumc.68.2.137.35952

Lim, Y.J., Ko, Y.G., Shin, H.C.y Cho, Y. (2013). Prevalence and Correlates of Complete Mental Health in the South Korean Adult Population. En C.L.M. Keyes (Ed.), Mental Well-Being (pp. 91-109). Netherlands: Springer.

Macdonald, C., Bore, M. y Munro, D. (2008). Values in action scale and the Big 5: An empirical indication of structure. Journal of Research in Personality, 42(4), 787-799. http:// dx.doi.org/10.1016/j.jrp.2007.10.003

Meyer, G.J., Finn, S.E., Eyde, L.D., Kay, G.G., Moreland, K.L., Dies, R.R., . . . Reed, G.M. (2001). Psychological testing and psychological assessment: A review of evidence and issues. American psychologist, 56(2), 128. 
Orcutt, H.K. (2006). The prospective relationship of interpersonal forgiveness and psychological distress symptoms among college women. Journal of Counseling Psychology, 53(3), 350-361. http://dx.doi.org/10.1037/00220167.53.3.350

Park, N. y Peterson, C. (2008). Positive Psychology and Character Strengths:Application to Strengths-Based School Counseling. Professional School Counseling, 12(2), 85-92. http:// dx.doi.org/10.5330/PSC.n.2010-12.85

Park, N. y Peterson, C. (2009). Character Strengths: Research and Practice Journal of College and Character, 10(4). http://dx.doi. org/10.2202/1940-1639.1042.

Peterson, C. (2006). The Values in Action (VIA) Classification of Strengths. In M. C. I. S. Csikszentmihalyi (Ed.), A life worth living: Contributions to positive psychology (pp. 29-48). New York, NY: Oxford University Press.

Peterson, C., Park, N. y Seligman, M.E.P. (2006). Greater strengths of character and recovery from illness. The Journal of Positive Psychology, 1(1), 17-26. http://dx.doi. org/10.1080/17439760500372739

Peterson, C. y Seligman, M.E.P. (2004). Character strengths and virtues: A handbook and classification. Washington, DC: American Psychological Association.

Petrillo, G., Capone, V., Caso, D. y Keyes, C.L.M. (2015). The Mental Health Continuum-Short Form (MHC-SF) as a Measure of WellBeing in the Italian Context. Social Indicators Research, 121(1), 291-312. http://dx.doi.org /10.1007/s11205-014-0629-3

Ruch, W., Proyer, R.T., Harzer, C., Park, N., Peterson, C.y Seligman, M.E.P. (2010). Values in Action Inventory of Strengths (VIA-IS). Journal of Individual Differences, 31(3), 138-149. http://dx.doi.org/10.1027/1614-0001/ a000022

Seligman, M.E.P. (2002). Positive psychology, positive prevention, and positive therapy. En C.R. Snyder y S. J. López (Eds.), Handbook of positive psychology (pp. 3-9). New York, NY: Oxford University Press.

Seligman, M.E.P., Steen, T.A., Park, N. y Peterson, C. (2005). Positive psychology progress: empirical validation of interventions. The American Psychologist, 60(5), 410-421. http://dx.doi. org/10.1037/0003-066X.60.5.410

Seligman, M.E.P., Rashid, T. y Parks, A.C. (2006). Positive Psychotherapy. American Psychologist, 61(8), 774-788. http://dx.doi. org/10.1037/0003-066X.61.8.774

Seligman, M.E.P. (2015). Chris Peterson's unfinished masterwork: The real mental illnesses. The Journal of Positive Psychology, 10(1), 3-6. http://dx.doi.org/10.1080/17439760.2014.888 582

Smith, T.B., McCullough, M.E. y Poll, J. (2003). Religiousness and depression: evidence for a main effect and the moderating influence of stressful life events. Psychological bulletin, 129(4), 614.

Theran, S.A. (2010). Authenticity with authority figures and peers: Girls' friendships, self-esteem, and depressive symptomatology. Journal of Social and Personal Relationships, 27(4), 519-534. http://dx.doi. org/10.1177/0265407510363429

Wood, A.M. y Tarrier, N. (2010). Positive Clinical Psychology: A new vision and strategy for integrated research and practice. Clinical Psychology Review, 30(7), 819-829. http://dx.doi. org/10.1016/j.cpr.2010.06.003

Yin, K., He, J. y Fu, Y. (2013). Positive Mental Health: Measurement, Prevalence, and Correlates in a Chinese Cultural Context. En C.L.M. Keyes (Ed.), Mental Well-Being (pp. 111-132). Netherlands: Springer. 
